\title{
UMA COMPARAÇÃO DE MÉTODOS DE PREVISÃO DE DEMANDA DE PEÇAS DE REPOSIÇÃO: UMA APLICAÇÃO AO TRANSPORTE FERROVIÁRIO
}

\section{A COMPARISON OF METHODS OF FORECASTING DEMAND FOR SPARES: AN APPLICATION TO THE RAIL}

\author{
Adelmo Inácio Bertolde ${ }^{1}$; Walter Paganucci Xavier Junior ${ }^{2}$ \\ ${ }^{1}$ Universidade Federal do Espírito Santo - UFES - Espírito Santo - ES - Brasil \\ adelmoib@gmail.com \\ ${ }^{2}$ Universidade Federal do Espírito Santo - UFES - Espírito Santo - ES - Brasil \\ walterpaganucci@hotmail.com
}

\begin{abstract}
Resumo
O objetivo principal deste trabalho é comparar o desempenho de modelos de previsão de demanda para peças de reposição utilizadas em manutenções, apresentando suas aplicações em dados de uma indústria real. A importância crescente do papel da manutenção no novo ambiente empresarial tem provocado o aumento da quantidade de pesquisas na área de modelagem e otimização da manutenção. Nesse contexto, este trabalho compara o desempenho de modelos de previsão de demanda para peças de reposição utilizadas em manutenções de motores a diesel, a partir de uma aplicação a um conjunto de dados de uma indústria real, que atua na área de transporte ferroviário. Os modelos experimentados foram Média Móvel, Suavização Exponencial Simples, Holt, Croston e ARIMA, além do método utilizado pela empresa estudada nesse trabalho, que tem o seu nome omitido. Após analisar uma grande amostra de peças de reposição, os resultados mostraram uma forte evidência de superioridade do modelo de Suavização Exponencial Simples em relação ao método de previsão utilizado pela empresa em questão. Este trabalho tem como principal contribuição apresentar a necessidade da utilização de métodos mais fundamentados na previsão de demanda de consumo de materiais na manutenção para que os impactos no nível de atendimento $e$ os custos na cadeia de suprimento possam ser controlados.
\end{abstract}

Palavras-chave: previsão de demanda; peças de reposição; motores a diesel; transporte ferroviário.

\section{Introdução}

Segundo Callegaro (2010), em face da importância crescente do papel da manutenção no novo ambiente empresarial, o interesse pela área de gerenciamento da manutenção vem crescendo ultimamente, com aumento do número de pesquisas na área de modelagem e otimização da manutenção. 
$\mathrm{O}$ aumento da quantidade de equipamentos de alta tecnologia nas indústrias se refletiu numa maior necessidade de peças de reposição para maximizar a utilização deste equipamento, logo, uma boa gestão de peças de reposição aumenta a produtividade, reduzindo o tempo ocioso do ativo e aumentando a utilização dos recursos.

A maioria dos trabalhos nessa área lida com políticas para assegurar o nível de estoque ideal, e só nos últimos anos a pesquisa começou a se concentrar em modelos de previsão para a demanda das peças de reposição na manutenção. Surgiu também outro aspecto: a importância de aumentar o valor da gestão dos processos de manutenção, contemplando aspectos técnicos, econômicos e organizacionais desta função (CALLEGARO, 2010). No entanto, existem muitas dificuldades na previsão da demanda de peças de reposição, isto porque as características deste tipo de material, tais como as expectativas elevada dos clientes para com os produtos, a demanda elevada de alguns itens e esporádica de outros, o aumento da complexidade dos produtos e a redução dos ciclos de vida dos mesmos diferem muito das de outros materiais da produção (COHEN; LEE, 1990; COHEN; ZHENG; AGRAWAL, 1997; MUCKSTADT, 2004; KUMAR, 2005 e, REGO, 2006 apud REGO; MESQUITA, 2011). Outro importante fator a ser considerado é a disposição única ou múltipla de locais de estocagem (KENNEDY; PATTERSON; FREDENDALL, 2002 apud REGO; MESQUITA, 2011). No caso deste trabalho, a aplicação foi feita num ambiente de único local de estocagem de peças.

De acordo com Cardoso (2006) em muitas organizações percebe-se um grande desvio da demanda prevista com o consumo real. Há um alto capital imobilizado em estoques de materiais, no entanto, algumas vezes é possível se observar a falta de algum material necessário para a manutenção. Diante de um cenário onde os ativos possuem valores elevados e os gastos com peças de reposição representam um grande percentual do total dos custos, novas práticas no processo de gestão da manutenção, aliados a uma metodologia de previsão fundamentada, são de grande importância para otimizar os custos e atingir a excelência em manutenção.

Este trabalho, por se tratar de um estudo de caso de caráter exploratório, não tem a pretenção de utilizar todas as técnicas e modelos disponíveis que remetem a previsão de demanda de peças mas, cabe ressaltar que, mesmo com o uso de modelos de previsão de demanda relativamente simples disponíveis na literatura, pode-se agregar ganhos que corroborem para uma boa gestão de estoque de peças de reposição.

O objetivo deste trabalho é o de comparar e identificar, dentre alguns métodos de previsão de demanda de peças de reposição, aqueles que otimizem os erros de previsão de consumo de peças na manutenção de motores a diesel de locomotivas utilizadas no transporte ferroviário. 


\section{Desenvolvimento}

\subsection{Características da manutenção}

No ciclo de vida de um sistema industrial, há a necessidade de trocar peças ou componentes. Quando uma quebra ocorre, acontece a intervenção da manutenção, que requer diversas ações, como por exemplo a etapa de fornecimento de peças de reposição. A duração desta fase é fortemente influenciada pela presença ou não dos materiais de reposição necessários no armazém de estoque da organização.

Tipicamente, as peças de reposição apresentam demanda esporádica seguida, eventualmente, por grandes intervalos de tempo sem demanda, tornando esta de difícil previsão. Ressalta-se ainda que a falta de peças pode gerar custos altos (HUA et al, 2007).

Callegaro (2010) ainda afirma que, a presença de tempos altos de indisponibilidade de equipamentos pode levar ao uso de componentes similares aos originais, podendo danificar o sistema. Além disso, normalmente, peças de reposição não são do tipo "uso geral" e por isso têm de ser empregadas somente para o uso e função para que foram compreendidas. Isso, inevitavelmente, esconde um grande risco de obsolescência, que ocorre quando acontece a substituição de um equipamento: o conjunto de peças de reposição que não são passíveis de reutilização em outros sistemas torna-se imediatamente obsoleto. As peças de reposição possuem, geralmente, um valor unitário elevado, o que exige esforços financeiros para a sua compra e também para a sua manutenção e armazenamento. Logo, peças de reposição têm características especiais que tornam a sua gestão altamente delicada e sofisticada.

Para uma avaliação destas características da demanda de peças de reposição, dois parâmetros reconhecidos no campo internacional são utilizados:

CV - Coeficiente de variação: desvio-padrão da demanda dividido pela demanda média e ADI intervalo médio de tempo entre duas demandas: razão entre a quantidade de períodos sem demanda dividido pela quantidade de períodos com demanda. Ghobbar et al (2003) sugerem alguns valores de corte para tais medidas que permitem uma caracterização mais detalhada da demanda de peças de reposição (ver Figura 1).

Figura 1 - Categorias de demanda de peças de reposição

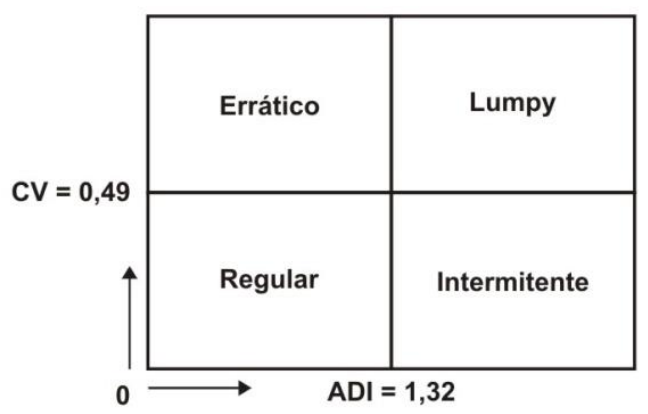

Fonte: Autoria própria 
Desta forma, quatro categorias podem ser definidas: Regular - peças de alto giro e baixo coeficiente de variação; Intermitente - peças com quantidade de períodos sem demanda (baixo giro); Errático - as que possuem alta variabilidade da quantidade solicitada, e possui alto giro, e Lumpy - é o mais difícil de controlar. Possui muitos períodos sem demanda (baixo giro) e uma grande variabilidade na quantidade. Esta divisão é importante para a investigação dos diferentes métodos de previsões, sendo parte essencial dos sistemas de gestão dos estoques (HUISKONEN, 2001 e BOYLAN; SYNTETOS; KARAKOSTAS, 2008).

\subsection{Custos da manutenção}

De acordo com Callegaro (2010), diferentes tipos de custos podem estar associados à gestão de peças de reposição. Primeiramente, há um custo financeiro significativo que envolve uma boa quantidade de capital para a compra (incluindo espaço utilizado, manutenção e, eventualmente, eliminação em caso de obsolescência). Um outro custo é o custo da falta do material. Concluindo, quando se fala em custo de gestão de peças de reposição dois aspectos contrastantes devem ser levados em consideração: o custo da falta e o custo de armazenamento. As fórmulas para calcular tais custos estão descritas em (1) e (2), respectivamente:

$$
C_{f}=P_{f} C_{h} T_{m} \frac{T}{T_{f}}
$$

sendo:

$C_{f}=$ Custo da falta do produto;

$P_{f}=$ Probabilidade de falta do produto;

$C_{h}=$ Custo por hora da falta de produto;

$T_{m}=$ Tempo médio entre reparos do produto;

$T_{f}=$ Tempo médio entre falhas;

$T=$ Intervalo de tempo considerado.

$$
C_{a}=R C \overline{S_{y}}
$$

sendo:

$C_{a}=$ Custo de armazenamento do produto;

$R=$ Custo médio de compra de uma peça de reposição;

$C=$ Custo de oportunidade do período para o capital imobilizado. 
$\bar{S}=$ Quantidade média de itens em estoque no período.

O objetivo da gestão de peças de reposição é encontrar o nível de estoque que minimize o custo total, que é função do custo de armazenamento e de falta. Chen et al (2009) relatam que se houvesse uma boa previsão das peças de reposição não haveria problema para controlar o nível de estoque.

\subsection{Previsão de demanda}

Mesmo assumindo que uma previsão exata é praticamente impossível, uma previsão que se aproxima da necessidade real pode fornecer um grande suporte para a tomada de decisões em uma organização (PADILHA, 2008). A previsão da demanda representa um dado fundamental para o planejamento empresarial, permitindo dimensionar adequadamente os recursos necessários à empresa (MAKRIDAKIS; WHEELWRIGHT; HYNDMAN, 1998 apud REGO; MESQUITA, 2011).

Um dos objetivos do setor da manutenção é gerir a incerteza da demanda de peças de reposição. Esta necessidade gerou desenvolvimento e levou a criação de alguns métodos e técnicas de previsão. Dentro dos métodos quantitativos de previsão encontram-se os modelos de séries temporais, que são, atualmente, largamente utilizados na previsão de demanda (PADILHA, 2008). Métodos estatísticos clássicos, como a suavização exponencial e análise de regressão, foram utilizados para tomar decisões durante várias décadas na previsão da demanda de peças de reposição.

Croston (1972) notou que métodos como a suavização exponencial não funcionavam de forma adequada para demandas irregulares, pois, o modelo colocava maior peso nas datas mais recentes da demanda. Para abordar esse viés o mesmo autor propôs um novo método de previsão de demanda que considerava o intervalo de tempo entre as demandas. Por outro lado, Syntetos e Boylan (2001) relataram um erro matemático no modelo de Croston e propuseram uma revisão para corrigílo, dando origem ao modelo SBA (Syntetos-Boylan Approximation). Syntetos e Boylan (2005) quantificaram o erro associado ao método de Croston (1972) e introduziram uma nova modificação envolvendo um fator aplicado ao modelo original de Croston (1972). Poucos anos após a introdução do método de Croston (1972), Box e Jenkins (1976) introduziram uma forma iterativa para gerenciar as previsões de peças de reposição com os modelos ARMA, ARIMA e S-ARIMA, métodos que hoje são amplamente utilizados.

Nas seções que seguem são apresentados, resumidamente, os modelos de previsão utilizados no presente trabalho.

\subsection{Modelos de previsão de demanda}

a) Média móvel: O método da Média Móvel consiste em calcular a média aritmética de uma série de n observações. A equação para a média móvel está descrita em (3): 


$$
F_{t}=\left(X_{t-1}+X_{t-2}+\ldots+X_{t-n}\right) / n
$$

onde:

$F_{t}=$ Previsão de demanda para o período $t$;

$X_{t-n}=$ Valor real da demanda no período $t-n$;

n=número de períodos (no presente trabalho foi utilizado $\mathrm{n}=12$ períodos).

b) Suavização exponencial simples: A suavização exponencial simples é um modelo muito popular quando se fala em séries temporais. Nada mais é do que uma média ponderada que atribui pesos decrescentes de forma exponencial para observações mais antigas. A equação de previsão (4) é a seguinte:

$$
F_{t+1}=\alpha \cdot X_{t}+(1-\alpha) \cdot F_{t}
$$

onde:

$F_{t+1}=$ Previsão de demanda para o período $t+1$;

$X_{t}=$ Valor real de demanda no período $t$;

$F_{t}=$ Previsão de demanda para o período $t ;$

$\alpha=$ Constante de suavização ( $\alpha$ pode assumir valores entre 0 e 1 , de forma que os desvios de previsão no período de ajuste do modelo seja mínimo).

c) Holt: O modelo de Holt (1957) descreve, de forma apropriada, dados em que se pode verificar a existência de tendência linear. Sua aplicação é válida pra séries não-estacionárias. As equações (5), (6) e (7) para a previsão são as seguintes:

$$
\begin{gathered}
A_{t}=\alpha \cdot X_{t}+(1-\alpha) \cdot\left(A_{t-1}+T_{t-1}\right), \\
T_{t}=\beta \cdot\left(A_{t}-A_{t-1}\right)+(1-\beta) \cdot T_{t-1}, \\
F_{t+p}=A_{t}+p \cdot T_{t},
\end{gathered}
$$

onde:

$F_{t+p}=$ Previsão de demanda para o período $t+p$;

$X_{t}=$ Valor real de demanda no período $t ;$

$A_{t}=$ Valor suavizado de $\mathrm{X}_{\mathrm{t}}$ no período $t$;

$T_{t}=$ Estimativa de tendência no período $t$;

$p=$ Períodos adiante de $t$ a serem estimados; 
$\alpha=$ Constante de suavização para os dados da série;

$\beta=$ Constante de suavização para a estimativa da tendência.

Morettin \& Toloi (2006) comentam que tal método é de fácil entendimento e adequado para séries com padrão de comportamento mais geral. Porém é limitado ao estudar as propriedades estatísticas como média e variância da previsão e, conseqüentemente, para a construção de um intervalo de confiança.

d) Croston: O modelo de Croston (1972) foi desenvolvido como uma opção para o modelo exponencial simples, com o objetivo de aplicar especificamente nos casos em que a demanda é intermitente. A previsão pelo método de Croston (1972) é dada pelas equações (8), (9) e (10):

$$
\begin{gathered}
Z_{t}=\alpha \cdot X_{t}+(1-\alpha) \cdot Z_{t-1}, \\
P_{t}=\alpha \cdot G_{t}+(1-\alpha) \cdot P_{t-1}, \\
F_{t+1}=Z_{t} / P_{t},
\end{gathered}
$$

onde:

$F_{t+1}=$ Previsão de demanda para o período $t+p$;

$Z_{t}=$ Valor suavizado da série no período $t$;

$P_{t}=$ Valor suavizado da série entre as transações "não zero";

$X_{t}=$ Valor "não zero" da série no período $t$;

$G_{t}=$ Intervalo de tempo entre as demandas "não zero";

$\alpha=$ Constante de suavização para os dados da série.

Syntetos e Boylan (2005) propuseram uma nova equação para as previsões, em que a equação de previsão da demanda é corrigida.

e) Modelo ARIMA: Os Modelos ARIMA são, em teoria, a classe mais geral de modelos para a previsão de uma série temporal. $\mathrm{O}$ ajuste fino de tal modelo consiste em acrescentar defasagens da série diferenciada e/ou defasagens dos erros de previsão para a equação de predição conforme necessário para remover qualquer vestígio de autocorrelação dos erros de previsão. A sigla ARIMA significa modelo "Auto-Regressivo Integrado de Médias Móveis".

Um modelo ARIMA é classificado como um “ARIMA (p,d,q)”, onde p é o número de termos autoregressivos, d é o número da ordem de diferenciação e q é o número de erros de previsões defasadas na equação de predição. Um modelo auto-regressivo integrado de médias móveis 
(ARIMA) é uma generalização do modelo ARMA, que por sua vez é uma combinação de um modelo AR e MA, onde p é a ordem de AR e q a ordem de MA.

Os critérios de seleção (CRIBARI, 2000) para modelos ARIMA mais utilizados são o AIC (Akaike Information criterion) e o AICC (Akaike Information Criterion Corrected). Esses critérios incorporam um termo de penalidade para o aumento do número de parâmetros (p e q) no modelo, de forma que modelos com o menor número de parâmetros sejam escolhidos. O critério de seleção escolhido para a identificação dos valores dos parâmetros p, d, q foi o AICC.

f) Modelo utilizado pela empresa estudada: A empresa em questão utiliza manutenções preventivas periódicas para manter seus ativos. Existem manutenções preventivas mais rápidas que acontecem com maior frequência, assim como, manutenções preventivas mais demoradas que acontecem com menor frequência. Nestas manutenções o ativo é inspecionado e algumas peças são substituídas. A substituição pode ser obrigatória ou condicional. O primeiro caso acontece quando a peça é substituída independentemente de sua condição. Já no segundo caso a peça é vistoriada e a sua substituição acontece devido às condições apresentadas. Outra forma de a peça ser substituída acontece nas manutenções corretivas. O ativo pode apresentar avarias e necessitar sofrer manutenção antes da manutenção preventiva programada.

Os cálculos utilizados pela empresa para identificar a necessidade de substituição de peças em cada manutenção são denominados de percentual de sucateamento. Este cálculo está ligado diretamente ao consumo de material nas manutenções passadas. O percentual de sucateamento é o fator de troca de peças em cada tipo de manutenção preventiva. Uma peça pode ter vários percentuais de sucateamento, de acordo com o tipo de plano em que está aplicada. O percentual de sucateamento é definido pela expressão (11) que segue:

$$
P_{s}=C M / T P P,
$$

onde:

$C M=$ Quantidade do material consumido em determinado plano;

$T P P=$ Soma dos planos preventivos executados (para peças definidas como troca obrigatória o percentual de sucateamento é considerado como 100\%).

O cálculo da previsão da demanda de materiais consiste em reunir as variáveis estudadas até o momento, gerando assim as previsões das quantidades de materiais necessárias para execução das atividades de manutenção indicadas. Para definir a demanda de materiais, a empresa utiliza-se de outra variável que é a quantidade de manutenções planejadas para os próximos meses. Com isso pode-se então definir o cálculo para definição da demanda de materiais por cada tipo de manutenção. A quantidade de materiais para execução das manutenções é definida pela expressão (12): 
onde:

$P M P P=$ Previsão do Material por Plano Preventivo;

$P S=$ Fator de troca de peças para cada tipo de manutenção;

$D M=$ Quantidade de manutenções a serem executadas em um período.

Além disso, a empresa também realiza uma previsão de forma qualitativa para o consumo de materiais nas manutenções corretivas. Desta forma, a previsão final de demanda de peças é a soma da previsão para manutenções corretivas com a previsão para as manutenções preventivas.

A despeito dos diversos trabalhos que tratam dos modelos de previsão de demanda, um artigo que merece destaque é o Rego e Mesquita (2011), que apresenta uma revisão da literatura sobre modelos de controle de estoques de peças de reposição, abordando tanto a previsão de demanda quanto o controle de estoques nos diferentes estágios do ciclo de vida das peças. Estes autores citam a carência de publicações de autores brasileiros sobre o tema. Kunigami e Osorio (2009) apresentaram um sistema para controle de peças em montagem de automóveis que apresentou considerável redução de estoques parados. Drohomeretski e Favaretto (2010) fazem um estudo exploratório sobre as causas e efeitos da falta de acuracidade nos estoques.

Convem destacar também os trabalhos: Ghobbar e Friend (2003), que constata a superioridade das técnicas média móvel ponderada, suavização exponencial dupla (Holt) e Croston num estudo sobre a demanda de peças de uma aeronave; de Willemain, Smart e Schwarz (2004), que aplica modelos de previsão para demandas intermitentes utilizando a técnica de reamostragem; Gutierrez, Solis e Mukhopadhyay (2008), que mostrou a superioridade de modelos com base em redes neurais sobre os métodos de suavização exponencial, Croston e SBA e, Teunter e Duncan (2009) que realizaram um estudo com peças de reposição para a Força Aérea Britânica, comparam os métodos de Média Móvel, Suavização Exponencial Simples, Croston, SBA e Reamostragem (bootstraping), verificando a superioridade das três últimas técnicas. O trabalho de Lopes Jaguaribe Pontes et al (2008), que embute o uso de séries temporais em sua construção, desenvolve um simulador que realiza projeções visando obter como resultados, por exemplo, a previsão de demanda e de reposição das peças. O simulador foi submetido a cenários diferentes e os resultados confirmaram a qualidade do sistema de simulação proposto. Já o trabalho de Pereira Salgado Junior et al (2010) apresenta um estudo de caso onde, fazendo uso de tecnologia da informação, gera melhorias no nível dos estoques e de serviço de uma empresa de bebidas carbonatadas. Leão e Dos Santos (2009) mostraram a eficácia do uso da ferramenta TRF (troca rápida de ferramentas), no contexto da manutenção, a fim de reduzir o tempo de máquina parada em uma siderurgia. Gomes e Wanke (2008) apresentam uma 
abordagem para gestão de estoques de peças de reposição com base em cadeias de Markov e modelagem via distribuição de Poisson, fazendo uma comparação com a simulação convencional.

\subsection{Medidas comparativas dos modelos}

Medidas de desvios de previsão são medidas utilizadas para comparações de diferentes métodos de previsão com o objetivo de determinar qual se aproxima mais da necessidade real. São os parâmetros na qual dois ou mais métodos de previsão são avaliados e comparados, em conexão com as demandas reais que ocorreram. No presente trabalho é utilizado como medida de desvio de previsão o Erro Quadrático Médio (EQM), para determinação do melhor modelo de previsão para cada peça de reposição. Desta forma, cada modelo teria um desvio EQM para cada um dos itens, e assim, os modelos poderiam ser comparados entre si para determinar qual o modelo com o melhor resultado (menor desvio o um menor valor do EQM) para cada item.

Além disso, foi criado um ranking para facilitar a visualização do desempenho de cada modelo para um mesmo item. Para cada item foi realizado um ranking, onde o modelo com menor desvio recebeu a primeira posição, o segundo com o menor desvio a segunda posição, e assim por diante até o modelo com maior desvio. Consequentemente, o pior resultado (ou modelo) para aquele item recebeu a sexta posição. Desta forma foi possível verificar qual o modelo que gerou as melhores previsões para determinado item, assim como o modelo que obteve as piores previsões.

\subsection{Banco de dados utilizado}

Os dados utilizados neste trabalho foram gerados a partir de um histórico de consumo de mais de 600 itens. utilizados na manutenção de motores a diesel, de uma empresa que atua no setor de transporte ferroviário. O histórico foi composto pela quantidade consumida em cada mês, para cada um dos itens, desde janeiro de 2008 até julho de 2011. Os dados em questão representam o consumo da manutenção (preventivas, periódicas e corretivas) realizado pela empresa.

Uma das limitações deste trabalho é que o histórico representa a quantidade do item que a oficina de manutenção retirou do armazém da empresa, não tendo este sido necessariamente consumido no mês que foi retirado do armazém. Isto acontece principalmente com itens de baixo preço e alto giro. Outra limitação ocorre quando a oficina solicita um item ao armazém mas ele não está disponível naquele momento. Um outro detalhe que vale ressaltar é que o histórico estudado foi fortemente impactado pela crise financeira mundial que ocorreu no ano de 2009, o que pode ter provocado a uma oscilação anormal na demanda de peças de reposição nesse período. No entanto, entende-se que essas limitações são pontuais e que não afeta o resultado do presente trabalho devido ao grande número de itens analisados. A Tabela 1 que segue apresenta a quantidade dos itens por classificação conforme as categorias de demanda descritas na Figura 1: 
Tabela 1 - Classificação conforme a categoria de demanda da peça

\begin{tabular}{cc}
\hline Classificação & Quantidade de peças \\
\hline Regular & 24 \\
Errático & 301 \\
Intermitente & 0 \\
Lumpy & 305 \\
\hline
\end{tabular}

Fonte: Autoria própria (2012)

A fim de medir a acurácia dos modelos de previsão, o histórico de 42 meses foi, primeiramente, dividido em 2 partes, "passado adotado" e "futuro adotado". A primeira parte, "passado adotado", foi de janeiro de 2008 até dezembro de 2010, totalizando 36 períodos. Esse histórico foi utilizado para que cada modelo se ajustasse à demanda, de forma que fossem encontrados os parâmetros que minimizassem os desvios entre o modelo e o histórico real. Já a segunda parte do histórico, "futuro adotado", composta pelos meses de janeiro de 2011 até julho de 2011, totalizando 6 meses, foi utilizada para testar as previsões geradas pelos modelos e medir os respectivos desvios.

Em todos os modelos duas considerações foram adotadas. Primeiramente todas as previsões do "futuro adotado" foram arredondadas para se tornarem números inteiros. Qualquer valor de previsão com parte decimal inferior a 0,5 era arredondado para baixo, e valores de previsão com parte decimal igual ou superior à 0,5 eram arredondados para cima. Outra consideração foi que não foram permitidos valores de previsão abaixo de zero, estes foram considerados como zero, ou seja, nenhum consumo previsto para aquele período. Na seção que se inicia são apresentados os resultados dos ajustes do modelo.

\section{Resultados e discussões}

Após o ajuste dos modelos apresentados na Seção 2.4, foi realizada uma análise comparativa dos modelos com base no valor do Erro Quadrático Médio (EQM) para cada modelo, independente do tipo de peça, ou seja, para a amostra completa (ver Tabela 2).

Tabela 2 - Mediana do EQM para cada modelo, amostra completa

\begin{tabular}{cc}
\hline Modelo & Mediana do EQM \\
\hline Empresa estudada & 57,5 \\
Suavização Exponencial & 34,3 \\
Média Móvel & 35,7 \\
Holt & 36,2 \\
Croston & 34,7 \\
ARIMA & 36,7 \\
\hline
\end{tabular}

Fonte: Autoria própria (2012)

A partir da Tabela 2, em uma primeira observação já pode-se notar que o modelo com o menor valor para a mediana do EQM é o modelo de Suavização Exponencial Simples, seguido pelo modelo de Croston. Por outro lado, verifica-se que o modelo com maior EQM, portanto, com maiores desvios de previsão, foi o modelo utilizado pela empresa estudada. 
Em seguida foram comparados os modelos entre si, para um mesmo item, através de um ranking, de forma a facilitar a visualização do desempenho de cada modelo. Na Tabela 3 é possível verificar a quantidade de vezes que cada modelo ficou em cada posição no ranking, que pode variar de 1 a 6 . Vale ressaltar que diferentes modelos puderam obter a mesma posição caso o EQM fosse igual. Se observada a média das posições de cada modelo, a constatação de superioridade do modelo de Suavização Exponencial Simples é novamente percebida. E, novamente, a inferioridade do modelo utilizado pela empresa estudada foi detectada. $\mathrm{O}$ modelo que mais vezes apareceu na primeira posição foi o modelo Croston, no entanto sua posição média foi superior a do modelo Suavização Exponencial Simples, que em $82 \%$ das vezes ficou entre os três melhores colocados e obteve uma posição média de apenas 2,22. Já o modelo utilizado pela empresa estudada impressiona pelo alto número de vezes que aparece na sexta colocação, terminando, desta forma, com uma posição de ranking média de 4,17, bem menor do que os demais modelos. A média das posições para os demais modelos Média Móvel, Holt, CROSTON e ARIMA ficaram bem próximas, com nenhum se destacando entre eles.

Tabela 3 - Ranking dos modelos para a amostra completa

\begin{tabular}{|c|c|c|c|c|c|c|c|}
\hline \multirow[t]{2}{*}{ Modelo } & \multicolumn{7}{|c|}{ Posição } \\
\hline & $\mathbf{1}^{\circ}$. & $2^{\circ}$. & $\mathbf{3}^{\mathbf{0}}$ & $4^{\circ}$. & $5^{\circ}$. & $6^{\circ}$. & Média do ranking \\
\hline Empresa estudada & 144 & 45 & 26 & 53 & 72 & 290 & 4,17 \\
\hline Suavização Exponencial & 243 & 166 & 109 & 68 & 37 & 7 & 2,22 \\
\hline Média Móvel & 208 & 132 & 99 & 86 & 75 & 30 & 2,65 \\
\hline Holt & 232 & 124 & 88 & 71 & 62 & 53 & 2,63 \\
\hline Croston & 257 & 107 & 63 & 56 & 77 & 70 & 2,68 \\
\hline ARIMA & 237 & 122 & 75 & 73 & 62 & 61 & 2,66 \\
\hline
\end{tabular}

Fonte: Autoria própria (2012)

A Tabela 4 apresenta os resultados dos desempenhos por categoria de peças, no caso, para itens Erráticos e Lumpy (não foram analizados os itens "Regular" e "Intermitente" devido ao pequeno número amostral - 24 para o primeiro caso e zero para o segundo caso). Para os itens Erráticos, o modelo de Croston, curiosamente, foi aquele que obteve os menores desvios. No entanto, o modelo de Suavização Exponencial Simples novamente obteve as melhores posições no ranking, com média igual a 2,54.

Tabela 4 - Mediana do EQM para cada modelo, itens erráticos e Lumpy

\begin{tabular}{ccccc}
\hline \multirow{2}{*}{ Modelo } & \multicolumn{2}{c}{ Itens erráticos } & \multicolumn{2}{c}{ Itens Lumpy } \\
\cline { 2 - 5 } & $\begin{array}{c}\text { Mediana do } \\
\text { EQM }\end{array}$ & $\begin{array}{c}\text { Posição no } \\
\text { ranking }\end{array}$ & $\begin{array}{c}\text { Mediana do } \\
\text { EQM }\end{array}$ & Posição no ranking \\
\hline Empresa estudada & 196,0 & 3,59 & 18,8 & 4,79 \\
Suavização Exponencial & 133,8 & 2,54 & 2,0 & 1,87 \\
Média Móvel & 131,0 & 3,08 & 2,0 & 2,21 \\
Holt & 136,0 & 2,91 & 2,5 & 2,24 \\
Croston & 126,2 & 3,06 & 2,3 & 2,19 \\
ARIMA & 141,5 & 3,07 & & \\
\hline
\end{tabular}

Fonte: Autoria própria (2012) 
Ainda a partir da Tabela 4 é possível notar que no caso dos 305 itens classificados como Lumpy (que são difíceis de controlar por possuirem baixo giro e uma grande variabilidade na quantidade) novamente verificou-se a superioridade do modelo de Suavização Exponencial Simples, tanto nas posições do ranking quanto nos desvios. Ressalta-se que, tanto para os itens Erráticos quanto para os itens Lumpy, o modelo da empresa estudada obteve os piores resultados quando comparados com todos os modelos. Ou seja, o modelo da empresa estudada obteve os resultados de previsão mais distantes da necessidade real.

\subsection{Análises de dispersão - CV e ADI}

Foi também realizada uma avaliação de cada modelo conforme a variação do CV (Coeficiente de Variação) e do ADI (Intervalo de Tempo Médio Entre Duas Demandas). Coletou-se, para cada modelo, os itens onde ele obteve o menor desvio (EQM) entre todos os modelos, ou seja, onde ele foi o mais preciso. Desta forma foi possível verificar para qual valores de CV e ADI o modelo se comportou com a maior precisão. Os resultados estão na Tabela 5 que segue.

Tabela 5 - Mediana de CV e ADI para os modelos com menores valores de EQM

\begin{tabular}{ccc}
\hline Modelo & \multicolumn{2}{c}{ Mediana } \\
\cline { 2 - 3 } & CV & ADI \\
\hline Empresa estudada & 1,26 & 0,50 \\
Suavização & 2,20 & 2,60 \\
Exponencial & 2,29 & 3,50 \\
Média Móvel & 2,05 & 2,27 \\
Holt & 1,97 & 2,27 \\
Croston & 2,03 & 2,27 \\
ARIMA & & \\
\hline
\end{tabular}

Fonte: Autoria própria (2012)

A partir da Tabela 5 foi possível verificar que o modelo da empresa estudada somente conseguiu atingir maior precisão que os demais modelos nos itens de baixo ADI e CV, ou seja, nos itens com maiores giros e menores variação na quantidade da demanda.

\section{Conclusões}

Este trabalho objetivou identificar, através de comparações, modelos de previsão de demanda para peças de reposição que aperfeiçoem a previsão de consumo na manutenção de motores a diesel de locomotivas de uma determinada empresa.

Este tema é de grande relevância no contexto do gerenciamento de estoque de peças, e, conforme, Callegaro (2010), é crescente o papel da manutenção no novo ambiente empresarial, ocasionando um aumento do número de pesquisas na área de modelagem, gerenciamento e otimização da manutenção.

A partir dos resultados obtidos neste trabalho foi possível verificar que o modelo com o menor valor para a mediana do Erro Quadrático Médio (EQM) é o modelo de Suavização 
Exponencial Simples, seguido pelo modelo de Croston. Por outro lado, verificou-se que o modelo com maior EQM, portanto, com maiores desvios de previsão, foi o modelo utilizado pela empresa estudada. Pode ser dito, em outras palavras, que o modelo utilizado pela empresa estudada obteve resultados inferiores, segundo o valor do EQM, à todos os demais modelos testados, obtendo melhores índices apenas para os grupos de peças com maior giro e com menor variação na quantidade demandada. Por outro lado, o modelo de Suavização Exponencial Simples foi o modelo que apresentou os melhores ajustes e, portanto, o que menor custo de reposição das peças.

Com relação à avaliação por categorias de peças, verificou-se, para quase todos os casos e categorias, que o modelo de Suavização Exponencial Simples obteve resultados superiores perante aos demais modelos, enquanto que o modelo da empresa estudada foi o modelo com os piores resultados. Os demais modelos Média Móvel, Holt, Croston e ARIMA obtiveram resultados relativamente próximos e ficaram, em todos os casos, posicionados entre o modelo de Suavização Exponencial Simples e o modelo da empresa estudada.

A análise a partir da divisão em categorias, utilizando CV e ADI, gerando as 4 categorias Regular, Errático, Intermitente e Lumpy, se mostrou de certa forma útil ao acentuar a desvantagem do uso do modelo da empresa estudada na previsão de demanda de peças.

É importante ressaltar que, ainda que neste trabalho se tenha feito uso de modelos relativamente simples, os mesmos já se mostraram superiores ao utilizado pela empresa estudada para a maioria dos casos analisados, no que tange a estimação da demanda de peças de reposição. Acredita-se, porém, que exista ainda espaço para evoluir no estudo de previsão de demanda, realizando outras análises com modelos que levem em consideração a sazonalidade ou técnicas que incomporem covariáveis, como por exemplo, a quilometragem percorrida e a quantidade de planos preventivos realizados e a serem realizados.

\begin{abstract}
The growing importance of the role of maintenance in the new business environment has led to an increased amount of research in modeling and optimization of maintenance. In this context, this paper compares the performance of models for forecasting demand for spare parts used for maintenance of diesel engines, from an application to a set of data from a real industry, which operates in the area of rail transport. The models were tested Moving Average, Simple Exponential Smoothing, Holt, Croston and ARIMA, and the method used by the company studied in this work, which bears his name withheld. After analyzing a large sample of spare parts, results showed strong evidence of superiority of model Single Exponential Smoothing over the forecasting method used by the company in question.
\end{abstract}

Key-words: demand forecasting; spare parts, diesel engines; transport rail. 


\section{Referências}

BOX, G. E. P. and JENKINS, G. M. Time Series Analysis: Forecasting and Control. San Francisco:Holden-Day. Revised Edition, 1976.

BOYLAN, J. E.; SYNTETOS, A. A.; KARAKOSTAS, G. C. Classification for forecasting and stock control: a case study. Journal of the Operational Research Society, v. 59, p. 473-481, 2008.

cross ref

CALLEGARO, A. Forecasting methods for spare parts demand. Universita Degli Studi di Padova, Pádua, 2010.

CARDOSO, M.V. Otimização da previsão de consumo de materiais na manutenção de material rodante. Monografia - Pontifícia Universidade Católica de Minas Gerais - Belo Horizonte, 2006.

CHEN, F.L.; CHEN, Y.C. An investigation of forecasting critical spare parts Requirement. World congress on computer science and information engineering, 2009.

COHEN, M. A.; LEE, H. L. Out of Touch with Customer Needs? Spare Parts and After Sales Service. Sloan Management Review, v. 31-2, p. 55-66, 1990.

COHEN, M. A.; ZHENG, Y. S.; AGRAWAL, V. Service parts logistics: a benchmark analysis. IIE Transactions, v. 29, p. 627-639, 1997.

cross ref

CRIBARI, F. N. Método de Previsão de Arrecadação Tributária. MIMEO, 2000.

CROSTON, J.D. Forecasting and stock control for intermittent demands. Operational Research Quarterly, n.23, 1972.

DROHOMERETSKI, Everton, FAVARETTO, Fábio. Um levantamento das causas e efeitos da falta de acuracidade nos estoques: um estudo exploratório. Revista Gestão Industrial. Ponta Grossa, v. 6, n. 2, 2010.

cross ref

GOMES, A. V. P. WANKE, P. Modelagem da gestão de estoques de peças de reposição através de cadeias de Markov. Gestão e Produção, São Carlos, v. 15, n. 1, abr. 2008.

GHOBBAR, A.A.; FRIEND, C.H. Evaluation of forecasting methods for intermittent parts demand in the field of aviation: a predictive model. Computers \& Operation Research, n.30, 2003.

GUTIERREZ, R. S.; SOLIS, A. O.; MUKHOPADHYAY, S. Lumpy demand forecasting using neural networks. International Journal of Production Economics, v. 111, p. 409-420, 2008.

cross ref

HOLT, C. C. Forecasting Seasonals and Trends by Exponentially Weighted Moving Averages. ONR Memorandum, v. 52, Pittsbugh: Carnegie Institute of Technology, 1957.

HUA, Z. S. et al. A new approach of forecasting intermittent demand for spare parts inventories in the process industries. Journal of the Operational Research Society, v. 58, p. 52-61, 2007.

cross ref

HUISKONEN, J. Maintenance spare parts logistics: Special characteristics and strategic choices. International Journal of Production Economics, v. 71, p. 125-133, 2001.

cross ref

KENNEDY, W. J.; PATTERSON, J. W.; FREDENDALL, L. D. An overview of recent literature on spare parts inventories. International Journal of Production Economics, v. 76-2, p. 201-215, 2002.

KUMAR, S. Parts Management Models and Applications. New York: Springer, 2005. 222 p. 
KUNIGAMI, F. J. OSORIO, W. R. Gestão no controle de estoque: estudo de caso em montadora aumobilística. Revista Gestão Industrial, Ponta Grossa, v. 5, n. 4, 2009.

cross ref

LEÃO, S. R. R. D. C. DOS SANTOS, M. J. Aplicação da troca rápida de ferramentas (TRF) em intervenções de manutenção preventiva. Revista Produção Online, Florianópolis, v. 9, n. 1, Mar. 2009.

LOPES JAGUARIBE PONTES, H. GUENKA PALMA, J. VIEIRA PORTO, A. J. SIMULINVE - Um simulador de inventário para um centro de distribuição de peças. Revista Produção Online, Florianópolis, v. 8, n. 3, out. 2008.

MAKRIDAKIS, S.G.; WHEELWRIGHT, S.C; HYNDMAN, R.J. Forecasting methods and applications. 3. ed. New York: John Willey \& Sons, 1998.

MORETTIN, P.A. e TOLOI, C.M.C. Análise de séries temporais. São Paulo: Edgard Blucher, 2006.

MUCKSTADT, J. A. Analysis and Algorithms for Service Parts Supply Chains. New York: Springer, 2004.277 p.

PADILHA, S.J. Metodologia para identificação de modelos de séries temporais na previsão da demanda de sobressalentes. Dissertação - Fundação Instituto Capixaba de Pesquisas em Contabilidade, Economia e Finanças, Vitória, 2008.

PEREIRA SALGADO JUNIOR, A. A tecnologia da informação como suporte ao ajuste da previsão da demanda: um estudo de caso em uma empresa de bebidas carbonatadas. Revista Produção Online, Florianópolis, v. 10, n. 3, p. 621648, ago. 2010 .

REGO, J. R. A Lacuna Entre a Teoria de Gestão de Estoques e a Prática Empresarial na Reposição de Peças em Concessionárias de Automóveis. Dissertação (Mestrado em Administração) - Faculdade de Economia, Administração e Contabilidade, Universidade de São Paulo - USP, 2006. 115 p.

REGO, J. R. do MESQUITA, M. A. de. Controle de estoque de peças de reposição em local único: uma revisão da literatura. PRODUÇÃ̃ [online]. 2011, vol.21, n.4, pp. 645-666. Epub 21-Jan-2011.

TEUNTER, R. H.; DUNCAN, L. Forecasting intermittent demand: a comparative study. Journal of the Operational Research Society, v. 60-3, p. 321-329, 2009.

cross ref

SYNTETOS, A.A.; BOYLAN, J.E. On the bias of intermittent demand estimates. International journal production economics, n.71, 2001.

SYNTETOS, A.A.; BOYLAN, J.E. The accuracy of intermittent demand estimates. International journal production economics, n.21, 2005.

WILLEMAIN, T. R.; SMART, C. N.; SCHWARZ, H. F. A new approach to forecasting intermittent demand for service parts inventories. International Journal of Forecasting, v. 20, p. 375-387, 2004.

\section{cross ref}

\section{Dados dos autores:}

\section{Nome completo: Adelmo Inácio Bertolde}

Filiação institucional: Departamento de Estatística da Universidade Federal do Espírito Santo

Função ou cargo ocupado: Professor doutor em estatística

Endereço completo para correspondência: Departamento de estatística da UFES/CCE/UFES. Av.

Fernando Ferrari , n: 514, Goiabeiras, Vitória - ES. 
Telefones para contato: (27) 4009-2481, ou (27) 9941-8575

e-mail: adelmoib@gmail.com

\section{Nome Completo: Walter Paganucci Xavier Junior}

Filiação Institucional: América Latina Logística - ALL / Formação: Engenheiro Civil - Universidade Federal do Espírito Santo - UFES

Função ou cargo ocupado: Gerente de Planejamento Financeiro

Endereço completo: Av. Presidente Affonso Camargo, 633, apto 502, Cristo Rei, Curitiba-PR CEP: 80050-370

Telefones para contato: (41) 9266-3091

e-mail: walterpaganucci@hotmail.com

Submetido em: 30/08/2012

Aceito em: 08/08/2013 